\title{
Analytical study of the energy rate balance equation for the magnetospheric storm-ring current
}

\author{
A. L. Clúa de Gonzalez, W. D. Gonzalez \\ INPE, CP 515, São José dos Campos, SP, Brasil \\ Received: 22 September 1997 / Revised: 18 March 1998 / Accepted: 25 March 1998
}

\begin{abstract}
We present some results of the analytical integration of the energy rate balance equation, assuming that the input energy rate is proportional to the azimuthal interplanetary electric field, $E_{y}$, and can be described by simple rectangular or triangular functions, as approximations to the frequently observed shapes of $E_{y}$, especially during the passage of magnetic clouds. The input function is also parametrized by a reconnection-transfer efficiency factor $\alpha$ (which is assumed to vary between 0.1 and 1 ). Our aim is to solve the balance equation and derive values for the decay parameter $\tau$ compatible with the observed Dst peak values. To facilitate the analytical integration we assume a constant value for $\tau$ through the main phase of the storm. The model is tested for two isolated and well-monitored intense storms. For these storms the analytical results are compared to those obtained by the numerical integration of the balance equation, based on the interplanetary data collected by the ISEE-3 satellite, with the $\tau$ values parametrized close to those obtained by the analytical study. From the best fit between this numerical integration and the observed Dst the most appropriate values of $\tau$ are then determined. Although we specifically focus on the main phase of the storms, this numerical integration has been also extended to the recovery phase by an independent adjust. The results of the best fit for the recovery phase show that the values of $\tau$ may differ drastically from those corresponding to the main phase. The values of the decay parameter for the main phase of each event, $\tau_{m}$, are found to be very sensitive to the adopted efficiency factor, $\alpha$, decreasing as this factor increases. For the recovery phase, which is characterized by very low values of the power input, the response function becomes almost independent of the value of $\alpha$ and the resulting values for the decay time parameter, $\tau_{r}$, do not vary greatly as $\alpha$ varies. As a consequence, the relative values of $\tau$ between the main
\end{abstract}

Correspondence to: A. L. Clúa de Gonzalez Fax: 55-12-345-6810; e-mail: alicia@dge.inpe.br and the recovery phase, $\tau_{m} / \tau_{r}$, can be greater or smaller than one as $\alpha$ varies from 0.1 to 1 .

\section{Introduction}

The energization of the ring current is usually described by a first order differential equation, known as the energy rate balance equation. Let $D$ be the pressure corrected Dst index,

$D=D s t-b \sqrt{p}+c$

where $p$ is the disturbed-day ram pressure of the solar wind, $\rho V^{2}$ ( $\rho$ and $V$ are respectively the solar wind density and velocity), $b$ is a constant of proportionality and $c$ gives the quiet-day contribution to $D$ (the corrected index should also incorporate a factor due to induced currents in the solid earth e.g., Langel and Estes, 1983; Stern, 1984, but this correction is usually neglected in the literature). After a transformation of the energy function to $D(t)$ times a constant factor in the energy rate balance equation (e.g. Gonzalez et al., 1989), this equation can be written as

$\frac{\mathrm{d} D(t)}{\mathrm{d} t}+\frac{D(t)}{\tau}=Q(t)$

where $Q(t)$ (given in unities of magnetic flux over time) is proportional to the input power for the energization of the ring current, and $\tau$ is the ring current decay time. Throughout this work we will assume, as many authors have done before, that most of the Dst variability represents the ring current evolution, although a recent work has questioned this classical assumption (Campbell, 1996).

The balance equation has been studied by many authors and commonly used values for the constants are $b=0.2 \mathrm{nT} /\left(\mathrm{eV} \mathrm{cm}^{-3}\right)^{1 / 2}$ (or about $16 \mathrm{nT} / \sqrt{\mathrm{nPa}}$ ) and 
$c=20 \mathrm{nT}$ (e.g., Burton etal., 1975; Akasofu, 1981; Feldstein et al., 1984; Pisarskij etal., 1989; Gonzalez etal., 1989; Feldstein, 1992). It describes the time evolution of the ring current associated function, $D$, as a function of the input, $Q$, and of the decay term $(D / \tau)$. Due to the difficulties involved in the exact knowledge of these factors, it has been customary to assume that they lie within a reasonable range of values and to solve the equation numerically, trying to get best fits between the observed and computed time evolution of the ring current energy intensity, which is proportional to the Dst index.

In the present work we follow an analytical approach to study the balance equation and to estimate a domain of values for the decay time compatible with the observed peak values of $|D|$. However, in order to make the equation analytically tractable some simplifying assumptions need to be completed. Among them, we have adopted simple expressions for the input functions and a constant value for the decay time during the main phase of the storm, $\tau_{m}$, as also has been frequently done by several authors (e.g., Burton et al., 1975; Feldstein et al., 1984). Nevertheless it is interesting to see that, regardless of such simplifying assumptions, and within the intrinsic limitations of the analytical method, the computed $D$ value resembles fairly well the observed one, at least for the events to which this approach has been applied (see Sect. 3 and the Appendix). Such results are encouraging in order to pursue an analytical approach such as the one explored here, at least with a similar importance to that given in the literature to more elaborate numerical techniques.

Diverse types of functions of the interplanetary plasma parameters have been postulated to represent the input function $Q$ (e.g., Gonzalez etal., 1989; Pisarskij et al., 1989). Basically, all of them depend on the amplitude of the negative $z$ component of the interplanetary magnetic field or southward field, $B_{s}$. In the present study we consider the input power as being proportional to the rectified azimuthal electric field, $E_{y}=\mathrm{v} B_{s} \quad\left(\mathrm{v}\right.$ is the solar-wind bulk velocity, and $B_{s}$ is equal to $B_{z}$ for negative $B_{z}$ and zero for positive $B_{z}$ ).

One possible way of solving Eq. (2) is to treat it as a linear system problem (e.g., Jenkins and Watts, 1968; Bendat and Piersol, 1971, 1980). The advantage of using this approach is merely formal, since it introduces in a natural way the optics of the linear filtering technique commonly used in this type of analysis (e.g., McPherron etal., 1986). As is known, the solution for a linear system (output function), in this case $D$, results from the convolution of the input signal, $Q$, with the impulse response function of the linear prediction filter. The latter is a characteristic of the system that, in this particular case, is an exponential decay function of time, a constant equal to $\tau$. The following result is obtained

$D(t)=\mathrm{e}^{-t / \tau}\left[D(0)+\int_{0}^{t} Q(z) \mathrm{e}^{z / \tau} \mathrm{d} z\right]$

where we have taken the origin of time as the starting moment of the energy-input impulse. In Eq. (3) we have disregarded the time lag that should normally appear in the output signal. However, since the effect of this lag is just a shifting in the ring-current response, there is no need to consider it in an explicit way.

In Sect. 2a, b we consider two simple cases for the input function, $Q$, that can be analytically integrated. They are the rectangular and the triangular input function cases. Then, in Sect. 3, we try to fit to one of these models two selected intense storms. In the Appendix we show the results of a numeric integration of Eq. (3) and the resulting $D(t)$ output for different values of $\tau$ are compared to the observed storm evolution, so that the input functions and the values of $\tau$ that best reproduce this evolution can be estimated.

\section{2a Response of $D(t)$ to a rectangular function}

Let us consider first the case for which the input function, $Q$, has a rectangular shape, with a negative constant value during a given time interval and is equal to zero outside it. This ideal input would resemble the limiting case for a disturbing interplanetary field that according to Gonzalez and Tsurutani (1987), is able to produce an intense storm, namely, a southward interplanetary magnetic field $B_{z}<-10 \mathrm{nT}$ acting over at least $3 \mathrm{~h}$. In general, we define this rectangular input as,

$Q(t)= \begin{cases}Q_{o}, & \text { if } 0 \leq t<T \\ 0 & \text { otherwise }\end{cases}$

where $Q_{o}$ is a negative constant and $T$ is the duration of the input.

In this case, a simple analytical result can be obtained for $D(t)$ from Eq. (3). For $t<T$, we have

$D(t)=\mathrm{e}^{-t / \tau} D(0)+Q_{o} \tau\left[1-\mathrm{e}^{-t / \tau}\right]$

and for $t \geq T$,

$D(t)=D(0) \mathrm{e}^{-t / \tau}+Q_{o} \tau\left[\mathrm{e}^{-(t-T) / \tau}-\mathrm{e}^{-t / \tau}\right]$.

Evidently, from the point of view of the geomagneticstorm evolution, Eq. (5a) corresponds to the main phase, while Eq. (5b) describes the recovery phase of the storm. In the case of a purely rectangular input function, the peak value of $D$ and characteristic times can also be easily computed. The peak value of $D$ occurs at the instant $t=t_{p}=T$ and is given by

$\left|D_{p}(\tau)\right|=|D(T)|=\left|\mathrm{e}^{-T / \tau} D(0)+Q_{o} \tau\left(1-\mathrm{e}^{-T / \tau}\right)\right|$

or its equivalent,

$\left|\frac{D_{p}(\tau)}{Q_{o}}\right|=|y(\tau)|=\mathrm{e}^{-T / \tau} y_{o}+\tau\left(1-\mathrm{e}^{-T / \tau}\right)$

where $\left.y_{(} \tau\right)=D_{p}(\tau) / Q_{o}$ and $y_{o}=D(0) / Q_{o}$.

Figure 1a, b gives two examples of the behavior of $D$ as a function of $t$, where $D(0)=0$, for two different sets of values of $T$ and $\tau$. The upper panels of these figures show the ideal rectangular input function, normalized to the $(0-1)$ range, while in the lower panels there are plots of the resulting values of $D(t) /\left|Q_{o}\right|$ versus $t$. In this figure 
we also show the following characteristic times: (1) $t_{p}$, interval of time for the development of main phase, (2) $w$, width at the half height of the storm evolution curve, (3) $w_{1}$, interval of time lasting from the moment in the main phase for which $|D(t)|$ reaches half the value corresponding to the peak $\left(\left|D_{p} / 2\right|\right)$, until the occurrence of the peak, and (4) $w_{2}$, time interval lasting from the occurrence of the peak and the moment during recovery phase for which $|D(t)|$ decreases to half of the value at the peak. For the rectangular function input these characteristic times are given by

$$
\begin{aligned}
& t_{p}=T, \\
& w=\tau \ln \left(1+\mathrm{e}^{T / \tau}\right), \\
& w_{1}=T+\tau \ln \left[\frac{1}{2}\left(\mathrm{e}^{-T / \tau}+1\right)\right], \\
& w_{2}=w-w_{1}=\tau \ln \left[\frac{2\left(1-\mathrm{e}^{-T / \tau}\right)}{\mathrm{e}^{-T / \tau}-1}\right]-T .
\end{aligned}
$$

From Fig 1a, b and Eqs. (6a), (6b) we can see that both the shape and the peak value of $D(t), D_{p}$, depend on the relative values of $T$ and $\tau$. For $\tau>T$, a sharper increase is observed in the computed $|D(t)|$ during the main phase, compared to the case with $\tau<T$. The behavior of the ratio $\left|D_{p} / Q_{o}\right|$, and characteristic times of the computed corrected index as a function of $\tau$, is shown in Fig. 2. The upper panel of Fig. 2 gives $|y(\tau)|$ (Eq. 6b, with the constant $y_{o}=0$, as derived from the assumed condition $D(0)=0$ ), while the lower panel gives $t_{p}, w$ and $w_{1}$ (Eq. $7 \mathrm{a}-\mathrm{c}$ ), also as functions of $\tau$. We discuss later how this type of curve can be used to find the value of $\tau$ for a given storm, once the ratio $\left|D_{p} / Q_{o}\right|$ is known. For this, however, some assumption about the input function, $Q$, has to be made, as shown in Sect. 3.

\section{2b Response of $D(t)$ to a triangular function}

The other simple case that we consider for the input energy is the triangular function defined as,
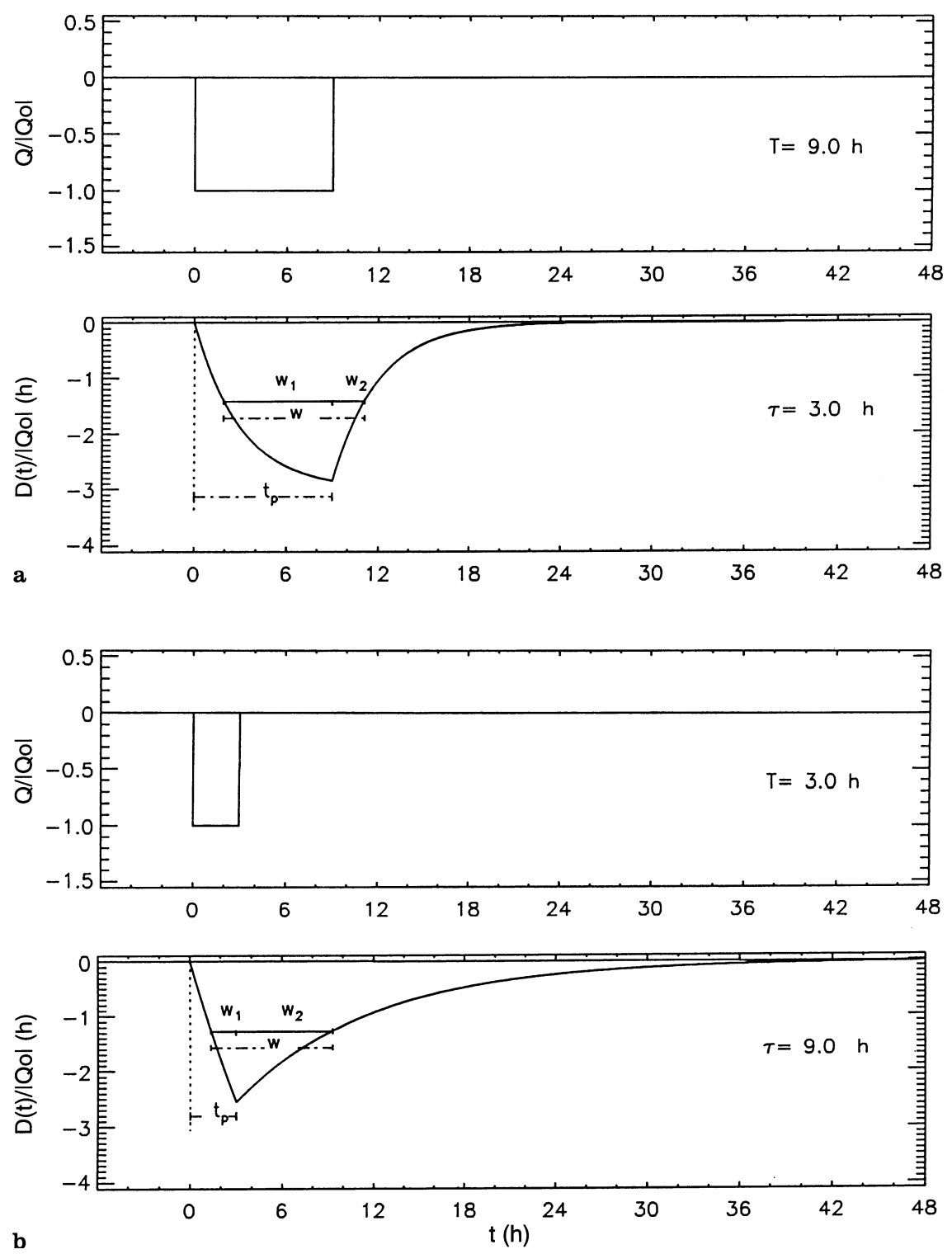

Fig. 1. a Behaviors of the rectangular normalized input function $Q(t) /\left|Q_{o}\right|$ according to Eq. (4) in the text (upper panel), and of the corresponding response function $D(t)$ also normalized in $\left|Q_{o}\right|$ according to Eq. $(5 \mathrm{a}, \mathrm{b})($ lower panel). The adopted values of $T$ and $\tau$ are, respectively 9 and 3 $\mathrm{h}$. The values of the characteristic times $t_{p}, w, w_{1}$ and $w_{2}$ (see text), are also shown in the plot. $\mathbf{b}$ The same as a, except for $T=3 \mathrm{~h}$ and $\tau=9 \mathrm{~h}$ 

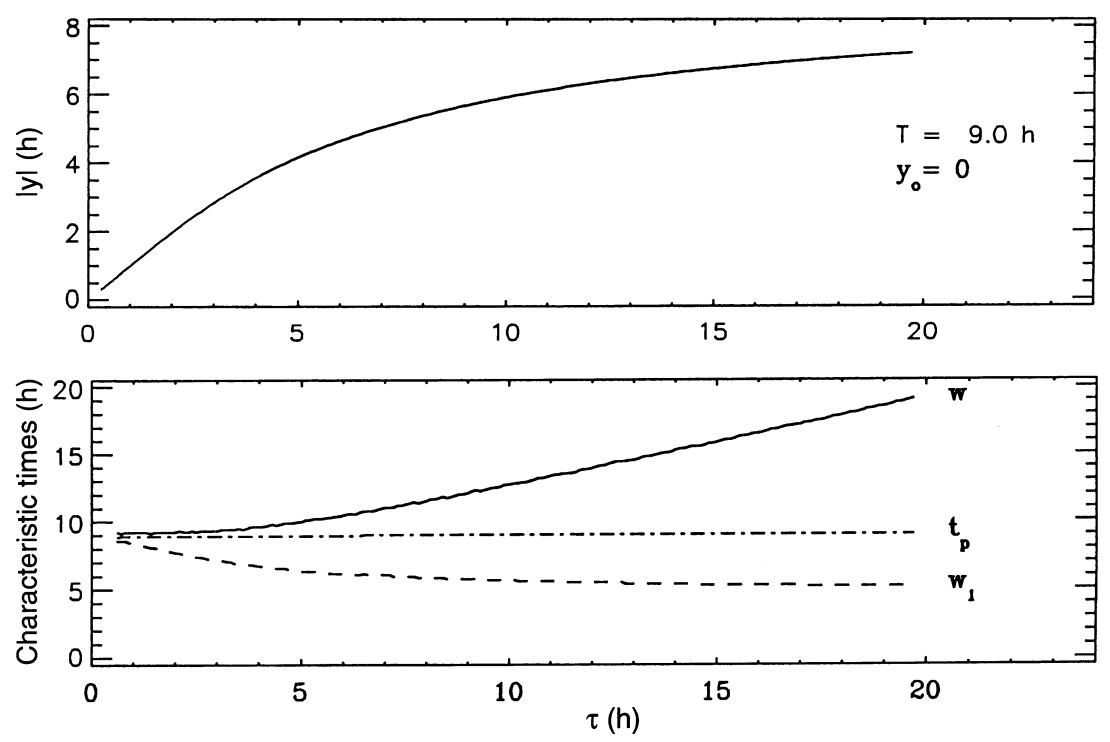

Fig. 2. Upper panel variation of the normalized peak value of $D(t),|y(\tau)|=\left|D_{p}(\tau) / Q_{o}\right|$, for the rectangular function of Fig. 1 as a function of $\tau$ (Eq. 6b), for $y_{o}=D(0) / Q_{o}=0$. Lower panel variation of the characteristic times $t_{p}$ (a constant for the particular case of a rectangular input function), $w$ and $w_{1}$, as functions of $\tau$

$Q(t)= \begin{cases}\frac{Q_{o}}{T_{1}} t, & \text { if } 0 \leq t<T_{1} \\ \frac{Q_{o}}{T_{2}}\left(T_{1}+T_{2}-t\right) & \text { if } T_{1} \leq t<T_{1}+T_{2} \\ 0 & \text { otherwise. }\end{cases}$

where, again, $Q_{o}$ is a negative constant.

With this expression for $Q(t)$, solution for Eq. (3) becomes,

for $0 \leq t<T_{1}$

$D(t)=D(0) \mathrm{e}^{-t / \tau}+Q_{o} \tau\left[\frac{t-\tau}{T_{1}}+\frac{\tau}{T_{1}} \mathrm{e}^{-t / \tau}\right]$

for $T_{1} \leq t<T_{1}+T_{2}$,

$$
\begin{aligned}
D(t)= & D(0) \mathrm{e}^{-t / \tau}+Q_{o} \tau\left[\frac{T_{1}+T_{2}+\tau-t}{T_{2}}\right. \\
& \left.+\frac{\tau}{T_{1}} \mathrm{e}^{-t / \tau}-\left(\frac{\tau}{T_{1}}+\frac{\tau}{T_{2}}\right) \mathrm{e}^{\left(T_{1}-t\right) / \tau}\right]
\end{aligned}
$$

and, for $t>T_{1}+T_{2}$,

$$
\begin{aligned}
D(t)= & D(0) \mathrm{e}^{-t / \tau}+Q_{o} \tau\left[\frac{\tau}{T_{1}} \mathrm{e}^{-t / \tau}\right. \\
& \left.-\left(\frac{\tau}{T_{1}}+\frac{\tau}{T_{2}}\right) \mathrm{e}^{\left(T_{1}-t\right) / \tau}+\frac{\tau}{T_{2}} \mathrm{e}^{\left(T_{1}+T_{2}-t\right) / \tau}\right] .
\end{aligned}
$$

An example of this type of input is given in Fig. 3. The upper panel of this figure shows the input function, defined by $T_{1}=3 \mathrm{~h}, T_{2}=7 \mathrm{~h}$. The lower panel shows the output function for $\tau=5 \mathrm{~h}$, according to Eqs. (9a)-(9c). The characteristic times $t_{p}, w, w_{1}$ and $w_{2}$ introduced in Sect. $2 \mathrm{a}$ are also shown in this plot. As observed in this figure, the analytical response for the ring current to a triangular input function is smoother than for the rectangular input, as expected from the fact that there is a smoother drop to zero of the input function for this case.

Due to the complexity of the curves given by Eq. $(9 \mathrm{a}-\mathrm{c})$, their peak values have been determined in a numerical way. Then, similar curves to those shown on Fig. 2 can be plotted for $|y(\tau)|=\left|D_{p}(\tau) / Q_{o}\right|$ and characteristic times. The only difference is that for this case $t_{p}$ is no longer a constant but varies slightly with $\tau$. The fact that the function $D_{p} / Q_{o}=f(\tau)$ is completely determined once the input is approximated by either the rectangular or triangular functions, has been the basis for the estimation of $\tau$ in this study. However, as discussed in the next section, the variable $Q_{o}$ is directly related to the efficiency in the energy transfer to the ring current and, since this efficiency is not known a priori, this parameter should be determined along with $\tau$.

\section{Analytical solution of the balance equation for two selected intense storms}

In this section we consider the analytical solution of the balance equation for two selected intense storms that occurred between August, 1978 to December, 1979, for which the ISEE-3 interplanetary data are available. The events are those corresponding to November 25-26, 1978, and March, 10-11, 1979, and constitute respective examples of the rectangular and the triangular approximations proposed in the previous sections. As mentioned, in order to predict the function $D(t)$ from the analytical integration of the energy balance equation, some assumption about the actual input power to the ring current, and consequently on the value of $Q$, must be made. According to Gonzalez et al. (1989), if the input power is assumed to be proportional to $E_{y}$, the following expression can be used for $Q$,

$Q \approx-\alpha \times 50\left[E_{y}\right] \mathrm{nT} / \mathrm{h}$

where $\left[E_{y}\right]$ is the numeric value of the modulus of the rectified electric field $\left|E_{y}\right|$ given in $\mathrm{mV} / \mathrm{m}$, and $\alpha$ is a factor of efficiency that has been estimated in previous studies to vary between 0.1 and 0.3 (Gonzalez and Mozer, 1974; Gonzalez et al., 1989). In this analysis it is assumed that $\alpha$ is parametrized with the values $0.1,0.2$, 0.3 and 1 . 

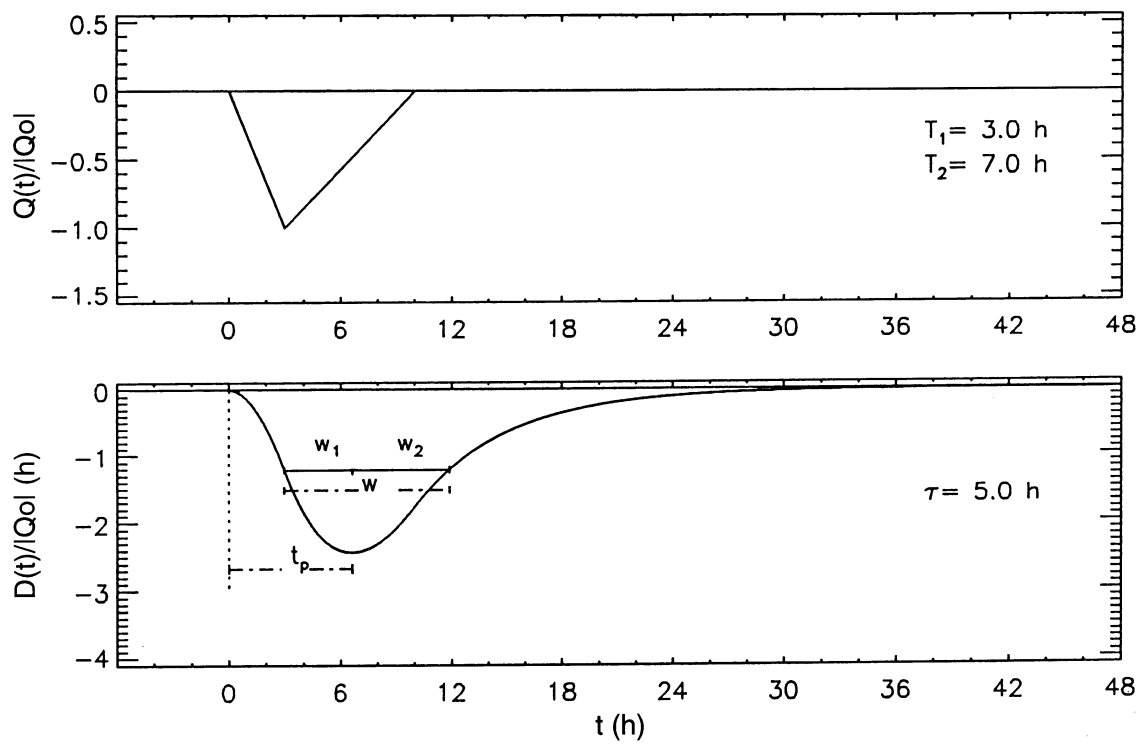

Fig. 3. The upper panel shows the triangular normalized input function, $Q(t) /\left|Q_{o}\right|$, obtained for $T_{1}=3 \mathrm{~h}$ and $T_{2}=7 \mathrm{~h}$ (Eq. 7). The lower panel shows the corresponding response function, also normalized in $\left|Q_{o}\right|$, for $\tau=5 \mathrm{~h}$. As in Fig. 1, the characteristic times $t_{p}, w, w_{1}$ and $w_{2}$, are shown in this plot

Table 1 summarily describes the main features of the two selected intense storms. The first and second columns of Table 1 give, respectively, the event identification and the main features of the observed $D(t)$. Since for both storms the levels of $D$ before and after the storm are approximately constant $\left(\approx D_{o}=D(0)\right)$, these values have been adopted as the zero level for each storm. $D_{p}$ is the absolute peak corresponding to each storm so that the effective peak of $D$ is given by $D_{p}-D_{o}$. The characteristic times $t_{p}, w_{1}$ and $w_{2}$ defined in Sect. 2, are also indicated. The third column shows the parameters for appropriate input functions (rectangular or triangular approximations), and the fourth column shows the peak values of $E_{y}, E_{y_{o}}$, which lead to the respective values of $Q_{o}$ in Eqs. (4) and (8) derived from Eq. (10).

After having modeled the input by one of the two analytical approximations, it is possible to plot $|y|=\left|D_{p} / Q_{o}\right|$ versus $\tau$ taking the efficiency factor $\alpha$ as a parameter. For the square function approximation, this relationship was computed from Eq. (6b), whereas for the triangular approximation a numeric computation was performed. Figures $4 \mathrm{a}$ and $5 \mathrm{a}$, which refer to event 1 (November 25-26, 1978) give an example of the approach used for rectangular approximation. The ISEE-3 data have a resolution of $5 \mathrm{~min}$ and the hourly $D s t$ values are similarly interpolated to $5 \mathrm{~min}$. The observed variations of $D(t)$ computed for each point from Eq. (1), with the constants $b$ and $c$ given in the introductory section, is shown by the light curve in the upper panel of Fig. 4a. In the lower panel of Fig. 4a the azimuthal electric field, $E_{y}=\mathrm{v} B_{s}$, computed from the ISEE-3 data is also given by the light curve. The analytical approximation of $E_{y}$, chosen as the rectangular function with $T=5.4 \mathrm{~h}$, is given by the heavy curve in the lower panel of Fig. 4a. Figure 5a shows the plot of $|y(\tau)|=\left|D_{p}(\tau) / Q_{o}\right|$ (Eq. (6b)) in the upper panel, and that of the characteristic times, $t_{p}, w$ and $w_{1}$ (Eqs. (7a)(7d)) in the lower panel, all as functions of $\tau$. Using Eq. (10) with $E_{y_{o}}=7 \mathrm{mV} / \mathrm{m}$ (see Table 1) and taking $\alpha=0.1$ and $\alpha=0.3$, as examples, two values for $Q_{o}$ are obtained. With these two values, one can enter at the

Table 1. Description of the events

\begin{tabular}{|c|c|c|c|}
\hline Event & $\begin{array}{l}\text { Storm } \\
\text { features }\end{array}$ & $\begin{array}{l}\text { Approximate } \\
\text { input function }\end{array}$ & $\begin{array}{l}E_{y_{o}} \\
{[\mathrm{mV} / \mathrm{m}]}\end{array}$ \\
\hline $\begin{array}{l}\text { November } 25-26,1978 \\
\text { (1) }\end{array}$ & $\begin{array}{l}D_{p}=-175( \pm 10) \mathrm{nT} \\
D_{o}=-50 \mathrm{nT} \\
t_{p}=5.7 \mathrm{~h} \\
w_{1}=3.3 \mathrm{~h} \\
w_{2}=6.4 \mathrm{~h}\end{array}$ & $\begin{array}{l}\text { Rectangular shape } \\
T=5.4 \mathrm{~h}\end{array}$ & $7( \pm 1)$ \\
\hline
\end{tabular}

In the first and second columns, the two events are identified and summarily described. $D_{p}$ is the value of the observed peak and $D_{o}$ is the approximate value of $D$ a few hours before and after the storm. $t_{p}, w_{1}$ and $w_{2}$ are the characteristic times defined in the text (notice that $\left.w=w_{1}+w_{2}\right)$. The third column describes the model adopted for each approximating function (see text). The fourth column gives the peak azimuthal electric field, $E_{y_{o}}$, for the assumed model and its estimated error 

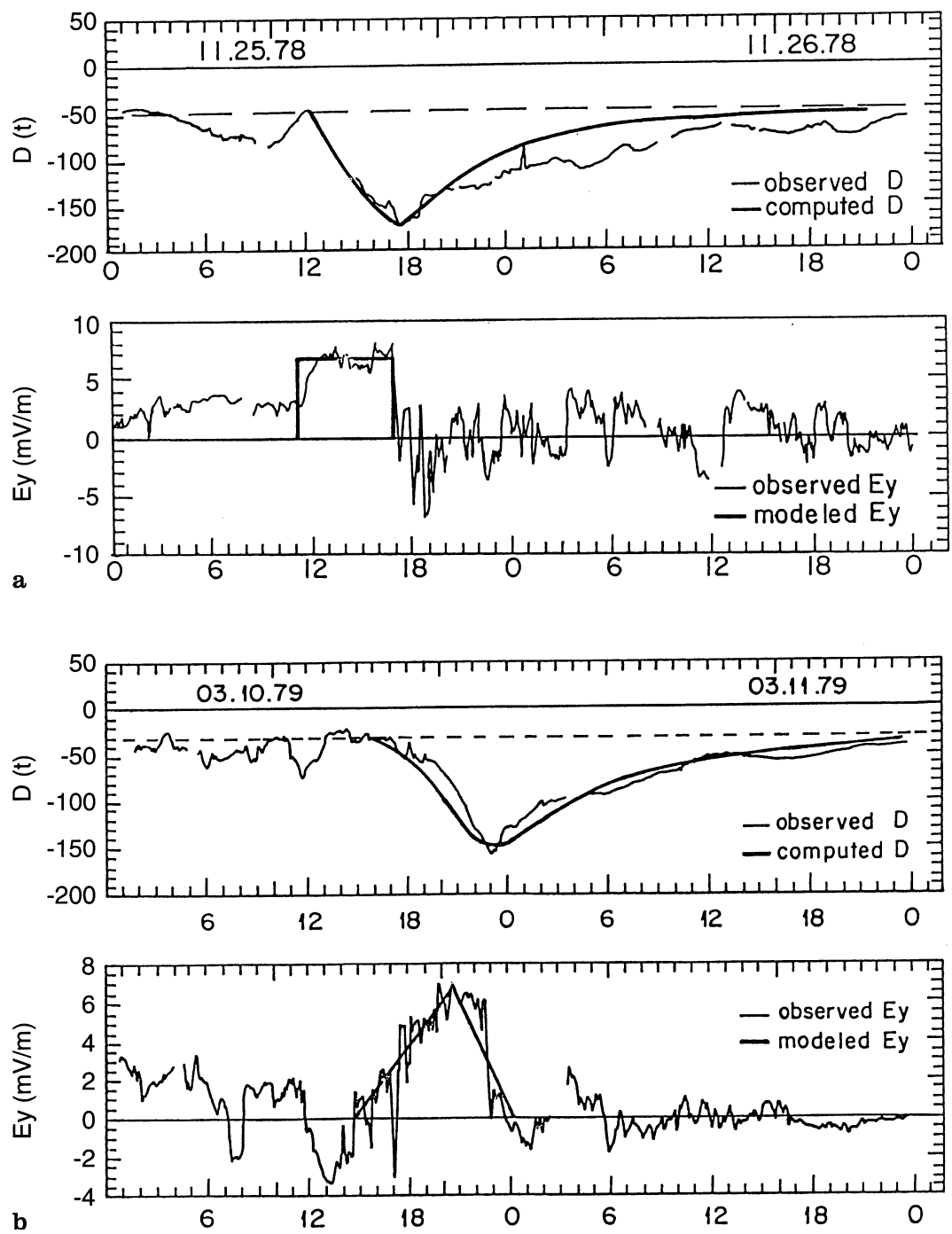

Fig. 4. a The intense geomagnetic storm of November 25-26, 1978. Lower panel: The light line gives the interplanetary azimuthal electric field, $E_{y}=\mathrm{v} B_{z}$, as inferred from the ISEE-3 data. The heavy line shows the rectangular approximation adopted for this event (Table 1). Upper panel: the light line gives the observed ram-pressure corrected $D s t$ index, $D(t)($ Eq. 1 with the values of $b$ and $c$ given in the text) and the heavy line is the result of the analytical approach based on the approximated rectangular function of the lower panel (see also Fig. 5a). The parameter $\alpha$ is assumed to be 0.1 and $\tau=6.3 \mathrm{~h}$ both for the main phase, and recovery phase. The dashed line gives the adopted zero level value for $D(t)$. b Similar to a but for the intense storm of March 10-11, 1979. For the computed $D$ curve (heavy line of the upper panel) $\alpha$ is also assumed to be 0.1 and two values of $\tau$ were selected, $\tau_{m}=14 \mathrm{~h}$ for the main phase and $\tau_{r}=8 \mathrm{~h}$ for the recovery phase ordinates of upper panel of Fig. 5a (dotted lines) and find that the corresponding values of $\tau$ are, respectively, 6.3 and $1.2 \mathrm{~h}$. In the same way, the lower panel of Fig. $5 \mathrm{a}$, allows estimation of the predicted values of the characteristic times for each case, but the validity of this will be discussed later. The curve computed through Eq. (6b) for the first case $(\alpha=0.1, \tau=6.3)$, is shown by the heavy line in the upper panel of Fig. 4a.

A completely similar approach has been made for the case of a triangular approximation using Eq. (9a-b). Figures $4 \mathrm{~b}$ and $5 \mathrm{~b}$, which correspond to event of March 10-11, 1979, exemplify this. The values adopted for $T_{1}$ and $T_{2}$ are respectively $6.2 \mathrm{~h}$ and $3.4 \mathrm{~h}$ (See Table 1 ). Considering again $\alpha=0.1$ and $\alpha=0.3$, the respective values of $14 \mathrm{~h}$ and $1.5 \mathrm{~h}$ can be obtained for $\tau$. We show the result obtained for $\alpha=0.1$ by the heavy line in the upper panel of Fig. 4b. However, we can see that for this event the fitting of the computed $D(t)$ improves using a different value of $\tau$ for the recovery phase (in the plot the adopted value for the recovery phase was $\tau_{r}=8 \mathrm{~h}$ ). As will be discussed later, the reason for this is that values of $\tau$ obtained by the analytical approach are basically derived for the main phase and do not necessarily adjust well for the recovery phase. In fact, as is shown by the numerical analysis of the Appendix, the values of $\tau$ for the recovery phase seem to change drastically compared to those of the main phase. The results obtained for the main phases of both events for $\alpha=0.1,0.2,0.3$ and 1 are summarized in Table 2. The values of $\tau$ and $w_{1}$ are given in hours. The numbers in parentheses are the errors in $\tau$, estimated according to the expression

$|\Delta \tau|=\frac{1}{|\mathrm{~d} y / \mathrm{d} \tau|}|\Delta y|$

where $|\Delta y|$ can be found from $\left|\frac{\Delta y}{y}\right|=\left|\frac{\Delta D_{p}}{D_{p}}\right|+\left|\frac{\Delta Q_{o}}{Q_{o}}\right|$ and $\mathrm{d} y / \mathrm{d} \tau$ can be numerically computed from the plot $y(\tau)$ versus $\tau$. Since the values of $\mathrm{d} y / \mathrm{d} \tau$ become smaller as $\tau$ increases, the errors increase with $\tau$.

It should be noticed that the use of some of the observed characteristic times, $t_{p}, w, w_{1}$ and $w_{2}$ $\left(=w-w_{1}\right)$, would provide additional equations to our equation system in order to find $\alpha$ and $\tau$ simultaneously. However, the following considerations apply. In first place, it is seen that the computed $w_{2}$, and consequently $w$, depend on the value of the decay time in the recovery 

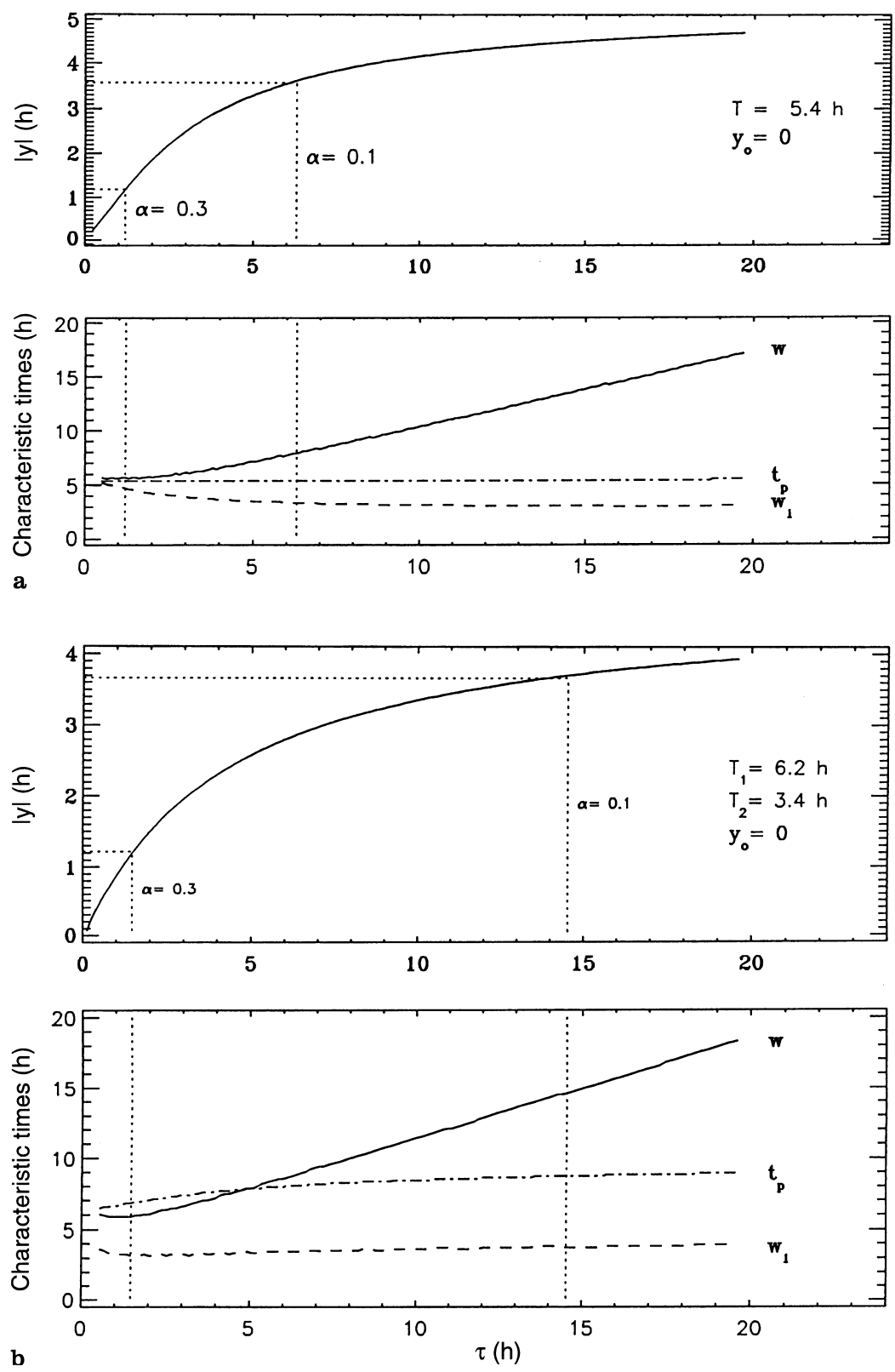

Fig. 5. a Like Fig. 2, this figure shows the variation of $|y(\tau)|=\left|D_{p}(\tau) / Q_{o}\right|$ (upper panel) and characteristic times (lower panel) as functions of $\tau$, but for a rectangular input function, with $T=5.4$ $\mathrm{h}$ (see Fig. 4a and Table 1). The horizontal dotted lines of the upper panel correspond to $y=3.57$ and $y=1.19$ derived for this event (Eq. 9), when $\alpha=0.1$ and $\alpha=0.3$ respectively. The inter-sections of these lines with the curve $|y(\tau)|$ give the roots $\tau=6.3 \mathrm{~h}$ and $\tau=1.2 \mathrm{~h}$. The inference of the corresponding characteristic times can be made from the curves of the lower panel. The practical meaning of this inference is discussed in Sect. 3 (see Table 2) b Similar to a but for event 2. The curves for this case are derived from the triangular approximation adopted for this event, with $T_{1}=6.2 \mathrm{~h}$ and $T_{2}=3.4 \mathrm{~h}$ (see Fig $4 \mathrm{~b}$ and Table 1). For $\alpha=0.1$ and $\alpha=0.3$ the respective roots found for the delay time are $\tau=14 \mathrm{~h}$ and $\tau=1.5$ h (see Table 2) phase, $\tau_{r}$, which can be different from the value of $\tau$ derived by the analytical model for the main phase. $t_{p}$ and $w_{1}$ in principle do not depend on the value $\tau_{r}$, but the fact that for large values of $\tau$ they tend to be independent of it (See Eq. (7b) and Fig. 2 for the rectangular input, and Fig. 5 for the triangular input), affects the procedure. Furthermore, the observed values for $w_{1}$ are very sensitive to the errors introduced by the lack of resolution in the Dst curve. In the case of event 1 , the comparison of the values of $w_{1}$ given in Tables 1 and 2, points to 0.1 as the most probable value of $\alpha$. Even though the situation is not so clear for event 2 , judging from the values of the associated standard deviations obtained in the Appendix (Table 3 ), $\alpha=0.1$ seems to give the best fit also for this case. However, due to the discussed uncertainties involved in the use of the characteristic times, we prefer to keep the results of Tables 2 and 3 (described in the Appendix) parametrized in $\alpha$.

\section{Summary and conclusions}

We present an analytical way of integrating the balance equation to obtain the variation of the pressure corrected Dst index, $D$, during intense geomagnetic storms (Eq. 2), based on two simplifying assumptions. One of the assumptions is that the ring-current decay time is constant during the main phase of the storm ( $\tau=\tau_{m}=$ const.), as has frequently been assumed in the literature, and the other that the input function of the equation, $Q(t)$, can be approximated by simple rectangular or triangular shapes. The input function is considered to be proportional to the rectified azimuthal electric field $E_{y}=\mathrm{v} B_{s}$, through an efficiency factor, $\alpha$, which has been parametrized as $\alpha=0.1,0.2,0.3,1.0$ (Eq. (10)). Under these assumptions it is possible to derive a unique function relating the ratio $D_{p} / Q_{o}$ (where $D_{p}$ and $Q_{o}$ are respectively the peak values of $D$ and $Q$ ) 
Table 2. Analytical results of the balance equation integration. The values of $\tau$ and $w_{1}$ that result from modeling the events of Table 1 , are given as a function of efficiency factor $\alpha$. The absolute errors in $\tau(\delta \tau)$ are derived from Eq. (11). All entries are in hours

\begin{tabular}{|c|c|c|c|c|c|c|c|c|}
\hline Event & \multicolumn{2}{|l|}{$\alpha=0.1$} & \multicolumn{2}{|l|}{$\alpha=0.2$} & \multicolumn{2}{|l|}{$\alpha=0.3$} & \multicolumn{2}{|l|}{$\alpha=1.0$} \\
\hline 2 & 14 (16) & 3.7 & $2.7(1.0)$ & 3.3 & $1.5(0.5)$ & 3.3 & $<0.3$ & - \\
\hline
\end{tabular}

Table 3. Best-fit results from the balance equation numerical integration (see Appendix). The decay times found for the main phase $\left(\tau=\tau_{m}\right)$ and for the recovery phase $\left(\tau_{r}\right)$ of the two events are given (in hours) for $\alpha=0.1,0.2$ and 0.3 . The columns labeled $\sigma_{m}$ and $\sigma_{r}$ give the respective standard deviation (in $\mathrm{nT}$ ) derived from Eq. (A1)

\begin{tabular}{|c|c|c|c|c|c|c|c|c|c|c|c|c|}
\hline \multirow[t]{2}{*}{ Event } & \multicolumn{4}{|c|}{$\alpha=0.1$} & \multicolumn{4}{|c|}{$\alpha=0.2$} & \multicolumn{4}{|c|}{$\alpha=0.3$} \\
\hline & $\tau_{m}(\mathrm{~h})$ & $\sigma_{m}(\mathrm{nT})$ & $\tau_{r}(\mathrm{~h})$ & $\sigma_{r}(\mathrm{nT})$ & $\tau_{m}(\mathrm{~h})$ & $\sigma_{m}(\mathrm{nT})$ & $\tau_{r}(\mathrm{~h})$ & $\sigma_{r}(\mathrm{nT})$ & $\tau_{m}(\mathrm{~h})$ & $\sigma_{m}(\mathrm{nT})$ & $\tau_{r}(\mathrm{~h})$ & $\sigma_{r}(\mathrm{nT})$ \\
\hline 2 & 14.0 & 6.7 & 7.0 & 9.0 & 2.7 & 10.4 & 6.0 & 10.6 & 1.7 & 21.9 & 5.0 & 8.6 \\
\hline
\end{tabular}

and $\tau$, for each value of $\alpha$. This function allows one to determine the expected $\tau$ for a given value of $\alpha$.

This method was used for two of the 10 intense storms in the interval covered by the ISEE-3 observations, namely those of November 25-26, 1978, and March 10-11, 1979, which look to be isolated events and do not have large data gaps. For these two events the rectangular and triangular approximations seem to be respectively appropriate. The values of $\tau$ for the main phase of these storms, $\tau_{m}$, were estimated in the way described in Sect. 3 (see Table 2). These estimations are taken as starting values for the fit presented in the Appendix, where instead of using the mentioned approximations, a numeric integration of the balance equation, based on the ISEE-3 data, is made. Varying $\tau_{m}$ in the neighbourhood of the previously obtained approximate value, different curves for the computed value of $D(t)$ were obtained. The criterium of a minimum standard deviation $(\sigma)$ of these curves from the observed corrected Dst index was used, to find the most probable values of $\tau$ as a function of the parameter $\alpha$. The values of $\tau_{m}$ and $\sigma_{m}$ resulting for the main phase of the storms, for $\alpha=0.1,0.2$ and 0.3 , are given in Table 3. Since, as seen in the previous section, the fit done for the main phase was not necessarily the best for the recovery phase, an independent adjustment was made for this phase, with the resulting values, $\tau_{r}$, shown also in Table 3 along with the respective standard deviations, $\sigma_{r}$.

The results obtained in the present analysis for the decay time parameter are limited by the assumption concerning the source of the energy rate input. This assumption is basically that this input is proportional to $E_{y}$, with a given efficiency factor $\alpha$ (Eq. 10). Under this hypothesis, and assuming that $\tau$ is constant during the main phase, it is possible to establish a threshold for the domain of $\tau$ such that the balance equation is satisfied. For the two cases considered, the computed values of $\tau_{m}$ vary from a few hours (for $\alpha=0.1$ ) to fractions of an hour (for $\alpha=1$ ). Orders of magnitude of several hours have been suggested by some authors (e.g. Burton et al., 1975; Feldstein et al., 1984; Pudovkin et al., 1985; Detman et al., 1994), but also of fractions of an hour by others (e.g. Vasyliunas, 1987; Gonzalez et al., 1989). Although in this study we cannot give a final answer in favor of one or the other alternative, the results tend to point towards the direction of $\alpha \approx 0.1$ and, consequently, to values of $\tau_{m}$ of the order of several hours. On the other hand, the adjustment done for the recovery phase, which is practically independent of $\alpha$, yields values of $\tau_{r}$ of the order of several hours, but with a less pronounced range of variability as compared to that corresponding to the values of $\tau$ for the main phase. As a consequence, depending on the adopted values of $\alpha, \tau_{r}$ can be smaller (for $\alpha \approx 0.1$ ) or larger (for $\alpha>0.1$ ) than $\tau_{m}$ (see Table 3 ).

The fact that a constant $\tau$ leads to a good fit for the main phase of the storms studied implies that a single loss mechanism would be operating, or at least be dominant, for the ring current during this phase. On the other hand, there seems to be a need to adopt a multiple valued $\tau$ to improve the fitting for the recovery phase, which indicates the presence of more than one loss mechanisms for this phase. This conclusion is in good agreement with those resulting from the loss processes that have been suggested in the literature for the two phases of the storm, involving mainly charge exchange for the main phase and charge exchange, cyclotron resonances and other dynamic losses for the recovery phase (e.g. Gonzalez et al., 1994; Vasyliunas, 1996). It should be said however that the hypothesis of a constant decay parameter for the main phase might be limited to the examples studied here, for which the main phase has a simple monotonic decrease. More complex events could present a more complex $\tau$ behavior.

Finally, it is interesting to point out that the simplified rectangular and triangular shapes for the input function used in this work could resemble the shapes typically associated with the $B_{s}$ structures of interplanetary magnetic clouds, which are known to be 


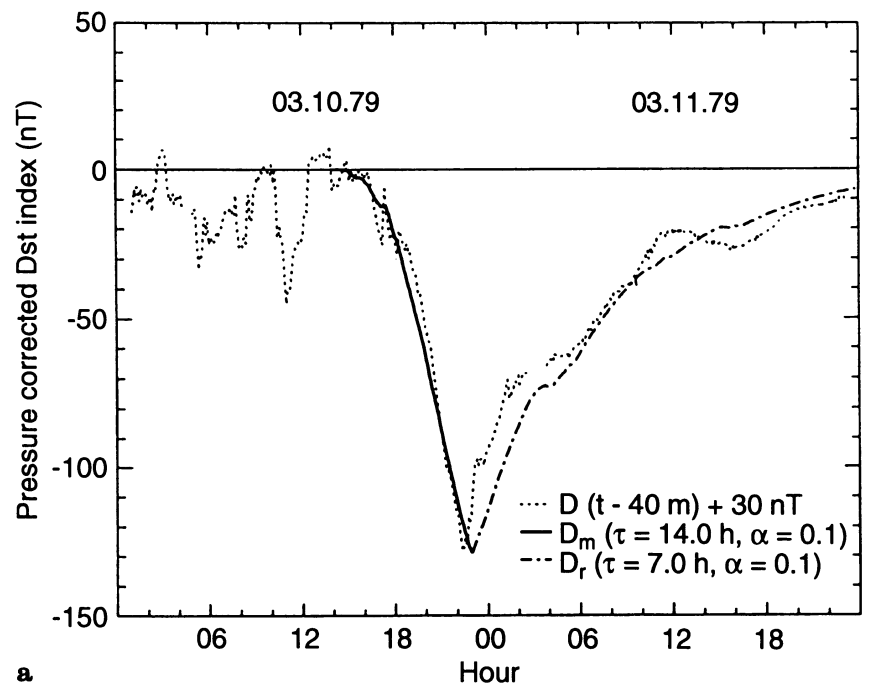

Fig. 6. a Numerical simulation of the response of $D(t)$ to the energy input given by Eq. (10) for event 2, for $\alpha=0.1$. The dotted line gives the computed values of $D(t)$, derived from $D s t$ and ISEE-3 data (Eq 1). The solid line $\left(D_{m}\right)$ is the result of the numerical integration of Eq. (3), starting at the time the storm began, for $D(0)=0$ and

very important causes of magnetic storms (e.g. Farrugia et al., 1997)

\section{Appendix}

\section{Numerical integration of the balance equation}

For the two events given in Table 1, characterized for having only small gaps and appearing to be isolated events, a numerical integration of Eq. (3) based on the ISEE-3 data has been done. We started using the values of $\tau_{m}$ obtained by the analytical solutions for each of these events (see Sect. 3 and Table 2). Then we varied them in small amounts around those values, computing for each case the variance given by

$\sigma^{2}=\frac{1}{m} \sum\left(D_{c}-D\right)^{2}$

where $D_{c}$ and $D$ are, respectively, the computed and the observed pressure corrected Dst index. Looking for those values of $\tau$ leading to a minimum of $\sigma^{2}$, we obtain the results (in hours) given in Table 3 , both for the main and the recovery phases (respectively called $\tau_{m}$ and $\tau_{r}$ ). Like in Table 2, the entries are parametrized by the efficiency factor $\alpha$. The values of the standard deviations obtained from Eq. A1 (in nT) are also given for each case.

Two examples of the performed fitting are given in Fig. 6a,b, that refer to event 2 , for $\alpha$ equal to 0.1 and 0.3 , respectively. In these figures the dotted line corresponds to the observed values for $D$, with the shifts in time and zero level indicated in the legend. The solid line $\left(D_{m}\right)$ is the result of the numerical integration of Eq. (3) starting at the beginning of the storm, with $D(0)=0$ and $\tau=\tau_{m}$.

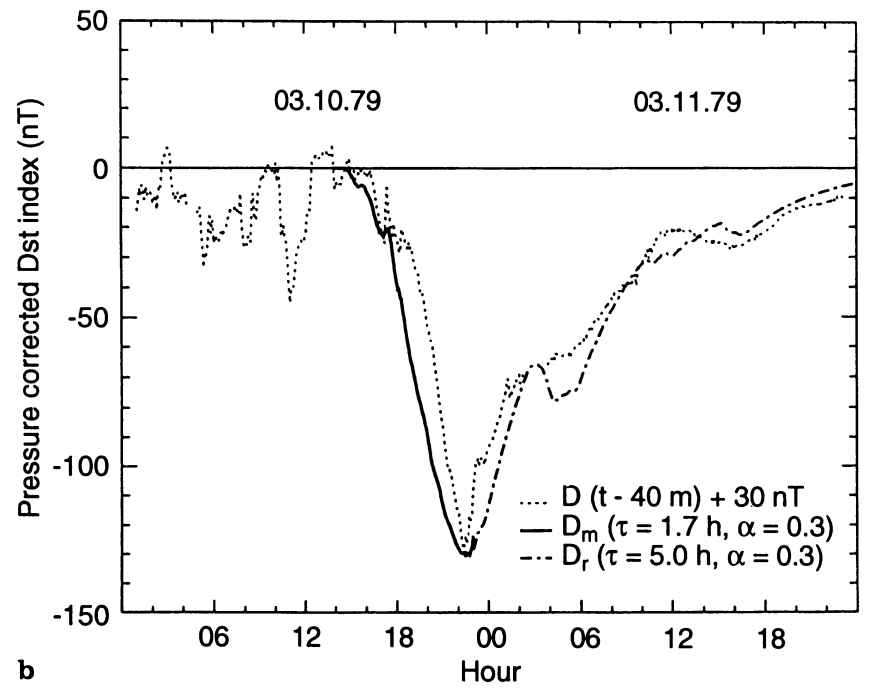

$\tau=\tau_{m}=14.0 \mathrm{~h}$. The dot-dashed line $\left(D_{r}\right)$ corresponds to the numerical integration of Eq. (3), starting at the peak for $D(0)=D_{p}$ and $\tau=\tau_{r}=7.0 \mathrm{~h}$. The respective shifts in time and zero level for $D(\mathrm{t})$ are also shown in the legends. b Same as a, except for $\alpha=0.3$, $\tau_{m}=1.7 \mathrm{~h}$ and $\tau_{r}=5.0 \mathrm{~h}$.

Finally, the dot-dashed line $\left(D_{r}\right)$ corresponds to the numerical integration of Eq. (3), starting at the time of the observed peak and with $D(0)=D_{p}$ and $\tau=\tau_{r}$.

Acknowledgements. This work was partially supported by the Fundo Nacional de Desenvolvimento Cientifíco e Tecnológico of Brazil. The authors would like to thank Dr. Bruce T. Tsurutani, from JPL, Pasadena, for the ISEE-3 data, and to Dr. Jo Ann Joselyn and Dr. T. R. Detman, from SEL/NOAA, for helpful discussions on this matter. A. L. C. G. benefited from a visiting fellowship from the Conselho Nacional de Pesquisa e Desenvolvimento, CNPq, of Brazil, at the National Oceanic and Atmospheric Administration, SEL, Boulder, CO.

Topical Editor K.-H. Glassmeier thanks I. A. Daglis and another referee for their help in evaluating this paper.

\section{References}

Akasofu, S.-I., Energy coupling between the solar wind and the magnetosphere, Space Sci. Rev., 28, 121-190, 1981.

Bendat, J. S., and A. G. Piersol, Random Data: Analysis and Measurements Procedures, Wiley-Interscience, New York, 1971.

Bendat, J. S., and A. G. Piersol, Engineering Applications of Correlation and Spectral Analysis, Wiley-Interscience, New York, 1980.

Burton, R. K., R. L. McPherron, and C. T. Russell, An empirical relationship between interplanetary conditions and Dst, J. Geophys. Res., 80(31), 4204-4214, 1975.

Campbell, W. H., Geomagnetic storms, the Dst ring-current myth and lognormal distributions, J. Atmos. Terr. Phys., 58(10), 1171-1187, 1996.

Detman, T. R., W. D. Gonzalez, and A. L. C. Gonzalez, Prediction of the geomagnetic (Dst) index by adaptive filtering of solar wind data, Proc. International Workshop on Artificial Intelligence Applications in Solar-Terrestrial Physics, Eds. J. A. Joselyn, H. Lundstedt and J. Trolinger, NOAA, SEL, Boulder, Colorado, pp. 159-165, 1994.

Farrugia, C. J., L. F. Burlaga, and R. P. Lepping, Magnetic clouds and the quiet-storm effect at the earth, in Magnetic Storms, Eds. 
B. T. Tsurutani, W. D. Gonzalez, Y. Kamide, and J. K. Arballo, American Geophysical Union Press, Washington D.C., Geophysical Monograph. Series 98, pp. 91-106, 1997.

Feldstein, Y. I., Modeling of the magnetic field of magnetospheric ring current as a function of interplanetary medium parameters, Space Sci. Rev., 59, 83-165, 1992.

Feldstein, Y. I., V. Y. Pisarskij, N. M. Rudneva, and A. Grafe, Ring current simulation in connection with interplanetary space conditions, Planet. Space Sci., 32(8), 975-984, 1984.

Gonzalez, W. D., F. S. Mozer, A quantitative model for the potential resulting from reconnection with an arbitrary interplanetary magnetic field. J. Geophys. Res., 79(28), 4186-4194, 1974.

Gonzalez, W. D., B. T. Tsurutani, Criteria of interplanetary parameters causing intense geomagnetic storms (Dst $<-100$ nT), Planet. Space Sci., 35(9), 1101-1109, 1987.

Gonzalez, W. D., B. T. Tsurutani, A. L. C. de Gonzalez, E. J. Smith, F. Tang, and S.-I. Akasofu, Solar wind-magnetosphere coupling during intense magnetic storms (1978-1979), J. Geophys. Res., 94(A7), 8835-8851, 1989.

Gonzalez, W. D., J. A. Joselyn, Y. Kamide, H. W. Kroehl, G. Rostoker, B. T. Tsurutani, V. M. Vasyliunas, What is a geomagnetic storm?, J. Geophys. Res, 99(A4), 5771-5792, 1994.
Jenkins, G. M., and D. G. Watts, Spectral Analysis and its Applications, Holden-Day, San Francisco, California, 1968.

Langel, R. A., and R. H. Estes, Large-scale, near-earth magnetic fields from external sources and the corresponding induced internal sources, NASA, Tech., Memo., TM-85012, 1983.

McPherron, R. L., D. N. Baker, L. F. Bargatse, "Linear filters as a method of real time predictions of geomagnetic activity", in Solar Wind-Magnetosphere Coupling, Eds. Y. Kamide and J. A. Slavin, Terra Scientific Publishing Company, Tokyo, pp 85-92, 1986.

Pisarskij, V. Y., Y. I. Feldstein, N. M. Rudneva, A. Prigancova, Ring current and interplanetary medium, Stud. Geophys. Geod., 33, 61-80, 1989.

Pudovkin, M. I., S. A. Zaitseva, and L. Z. Sizova, Growth rate and decay of magnetospheric ring current, Planet. Space Sci., 33(10), 1097-1102, 1985.

Stern, D. P., Energetics of the magnetosphere, Space Sci. Rev., 39, 1984.

Vasyliunas V. M., A method for evaluating the total magnetospheric energy output independently of the $\epsilon$ parameter, Geophys. Res. Lett., 14(12), 1183-1186, 1987.

Vasyliunas V. M., Global energetics of the magnetosphere during magnetic storms, Invited review paper, Chapman Conference on Magnetic Storms, Pasadena, California, February 12-16, 1996. 